\title{
Christoph Schmoeger
}

\section{COMMUTATIVITY UP TO A FACTOR IN BANACH ALGEBRAS}

\footnotetext{
Abstract. In this note we explore commutativity up to a factor $a b=\lambda b a$ for Hermitian or normal elements of a complex Banach algebra. Our results generalize results obtained for bounded linear operators on Hilbert spaces.
}

\section{Introduction}

The following result is due to Brooke, Busch and Pearson [3] (see also [6]). THEOREM 1.1. Let $A, B$ be bounded linear operators on a complex Hilbert space such that $A B=\lambda B A \neq 0(\lambda \in \mathbb{C})$. Then

(a) if $A$ or $B$ is self-adjoint, then $\lambda \in \mathbb{R}$;

(b) if both $A$ and $B$ are self-adjoint, then $\lambda \in\{-1,1\}$;

(c) if $A$ and $B$ are self-adjoint and one of them is positive, then $\lambda=1$.

The aim of this note is a generalization of Theorem 1.1 to Hermitian or normal elements of a complex Banach algebra.

Throughout, $\mathcal{A}$ will be a complex Banach algebra with unit $e$. The spectrum of an element $a \in \mathcal{A}$ will be denoted by $\sigma(a)$ and the spectral radius of $a$ by $r(a)$. Let $\mathcal{A}^{\prime}$ denote the dual space of $\mathcal{A}$. The numerical range $V(a)$ is the set

$$
V(a)=\left\{f(a): f \in \mathcal{A}^{\prime}, \quad\|f\|=f(e)=1\right\} .
$$

Proposition 1.2. Let $a, b \in \mathcal{A}$. Then

(a) $r(a b)=r(b a)$;

(b) $\sigma(a b) \backslash\{0\}=\sigma(b a) \backslash\{0\}$;

(c) if $a b=b a$, then $\sigma(a b) \subseteq \sigma(a) \sigma(b)$;

(d) $\sigma(a) \subseteq V(a)$.

Proof. (a), (b) und (c): $[5, \S 10, \S 11]$. (d) is shown in [2, Theorem 2.6]. 
We say that $a, b \in \mathcal{A}$ commute up to a factor if there is $\lambda \in \mathbb{C} \backslash\{0\}$ such that $a b=\lambda b a$. Our first result is an immediate consequence of Proposition $1.2(\mathrm{a})$.

Proposition 1.3. If $a, b \in \mathcal{A}, r(a b) \neq 0, \lambda \in \mathbb{C}$ and $a b=\lambda b a$, then $|\lambda|=1$.

EXAMPLE. This example shows that if $r(a b)=0$, then any value of $\lambda \neq 0$ may occur.

Let $\mathcal{A}=\mathbb{C}^{3 \times 3}, \lambda \neq 0, a=\left(\begin{array}{ccc}1 & 0 & 0 \\ 1 & \lambda & 0 \\ 1 & \lambda & \lambda^{2}\end{array}\right)$ and $b=\left(\begin{array}{lll}0 & 0 & 0 \\ 1 & 0 & 0 \\ 0 & 1 & 0\end{array}\right)$.

Then $a b$ is nilpotent and $a b=\lambda b a$.

An element $a \in \mathcal{A}$ is said to be Hermitian if $V(a) \subseteq \mathbb{R}$. We denote the set of all Hermitian elements of $\mathcal{A}$ by $H(\mathcal{A})$. From Proposition 1.2(d) we see that $\sigma(a) \subseteq \mathbb{R}$ if $a \in H(\mathcal{A})$. It is well-known (see [2, Example 5.3]), that if $\mathcal{A}$ is a $B^{*}$-algebra, then $a \in H(\mathcal{A})$ if and only if $a^{*}=a$.

Our next proposition collects the basic properties of Hermitian elements. For proofs see [2].

Proposition 1.4. (a) $a \in H(\mathcal{A}) \Leftrightarrow \| \exp ($ ita $) \|=1$ for all $t \in \mathbb{R}$;

(b) $H(\mathcal{A})$ is a closed real linear subspace of $\mathcal{A}$,

(c) $H(\mathcal{A}) \cap i H(\mathcal{A})=\{0\}$;

(d) if $a, b \in H(\mathcal{A})$, then $i(a b-b a) \in H(\mathcal{A})$;

(e) if $a \in H(\mathcal{A})$, then $r(a)=\|a\|$ (Sinclair's Theorem);

(f) if $a \in H(\mathcal{A})$, it does not follow that $a^{2} \in H(\mathcal{A})$.

Let $J(\mathcal{A})=\{h+i k: h, k \in H(\mathcal{A})\}$. It follows from Proposition 1.4(c) that each element of $J(\mathcal{A})$ has a unique representation of the form $h+i k$ with $h, k \in H(\mathcal{A})$. Therefore we may define a mapping * from $J(\mathcal{A})$ into itself by

$$
(h+i k)^{*}=h-i k \quad(h, k \in H(\mathcal{A})) .
$$

It is easy to verify that ${ }^{*}$ is a linear involution on $J(\mathcal{A})$.

We say that $a \in \mathcal{A}$ is normal if $a=h+i k$ with $h, k \in H(\mathcal{A})$ and $h k=k h$. Observe that $a \in \mathcal{A}$ is normal if and only if $a \in J(\mathcal{A})$ and $a a^{*}=a^{*} a$. An element $a \in \mathcal{A}$ is positive if $V(a) \subseteq[0, \infty)$. By [2, Theorem 5.14], we have

$$
a \in \mathcal{A} \text { is positive } \Leftrightarrow a \in H(\mathcal{A}) \text { and } \sigma(a) \subseteq[0, \infty) \text {. }
$$

\section{Results}

We begin with a generalization of Fuglede's theorem (see [1, Theorem 1]). For the convenience of the reader we include a proof, which is an adaption of Rosenblum's proof of the Putnam-Fuglede theorem (see [5, Theorem 12.16]). 
Proposition 2.1. Assume that $a, b, c \in \mathcal{A}, a, c$ are normal and that $a b=b c$. Then $a^{*} b=b c^{*}$.

Proof. By induction we have $a^{k} b=b c^{k}$ for $k=1,2,3, \ldots$ Therefore $\exp (a) b=b \exp (c)$, thus

$$
b=\exp (-a) b \exp (c) \text {. }
$$

Put $u_{1}=\exp \left(a^{*}-a\right)$ and $u_{2}=\exp \left(c-c^{*}\right)$. There are $h, k \in H(\mathcal{A})$ with $a=h+i k$ and $h k=k h$. Then $a^{*}-a=-2 i k$. Since $k \in H(\mathcal{A})$, Proposition 1.4(a) shows that $\left\|u_{1}\right\|=\|\exp (-2 i k)\|=1$. A similar argument gives $\left\|u_{2}\right\|=1$. From $a^{*} a=a a^{*}$ and $c c^{*}=c^{*} c$ we derive

$$
u_{1}=\exp \left(a^{*}\right) \exp (-a) \text { and } u_{2}=\exp (c) \exp \left(-c^{*}\right)
$$

hence, by (1),

$$
u_{1} b u_{2}=\exp \left(a^{*}\right) b \exp \left(-c^{*}\right)
$$

thus

$$
\left\|\exp \left(a^{*}\right) b \exp \left(-c^{*}\right)\right\|=\left\|u_{1} b u_{2}\right\| \leq\|b\| .
$$

Now define the entire function $f: \mathbb{C} \rightarrow \mathcal{A}$ by

$$
f(z)=\exp \left(z a^{*}\right) b \exp \left(-z c^{*}\right)
$$

Since the hypotheses of the proposition hold with $\bar{z} a$ and $\bar{z} c$ in place of $a$ and $c$, (2) implies that $\|f(z)\| \leq\|b\|$ for all $z \in \mathbb{C}$. By Liouville's theorem, $f(z)=f(0)=b$ for all $z \in \mathbb{C}$. Hence

$$
\exp \left(z a^{*}\right) b=b \exp \left(z c^{*}\right) \quad(z \in \mathbb{C}) .
$$

Thus

$$
\sum_{n=0}^{\infty} \frac{z^{n}}{n !}\left(a^{*}\right)^{n} b=\sum_{n=0}^{\infty} \frac{z^{n}}{n !} b\left(c^{*}\right)^{n} \quad(z \in \mathbb{C}) .
$$

Comparing coefficients gives $\left(a^{*}\right)^{n} b=b\left(c^{*}\right)^{n}$ for $n=0,1,2, \ldots$, hence $a^{*} b=b c^{*}$.

Corollary 2.2. If $a, b, c \in \mathcal{A}, a, c \in H(\mathcal{A}), \lambda \in \mathbb{C}$ and $a b=\lambda b c \neq 0$, then $\lambda \in \mathbb{R}$.

Proof. By_Proposition 2.1, $a^{*} b=\bar{\lambda} b c^{*}$. Since $a=a^{*}$ and $c=c^{*}, \lambda b c=$ $a b=a^{*} b=\bar{\lambda} b c^{*}=\bar{\lambda} b c$, thus $(\lambda-\bar{\lambda}) b c=0$, hence $\lambda=\bar{\lambda}$.

Proposition 2.3. Let $a \in H(\mathcal{A}), x \in \mathcal{A}$ and $a^{2} x=0$. Then $a x=0$.

Proof. We can assume that $\|x\|=1$. Then, by Proposition 1.4(a), for all $t \in \mathbb{R}$

$$
\begin{aligned}
1=\|x\| & =\|\exp (-i t a) \exp (i t a) x\| \leq\|\exp (-i t a)\|\|\exp (i t a) x\| \\
& =\| \exp (\text { ita } x\|\leq\| \exp (i t a)\|\| x\|=\| x \|=1
\end{aligned}
$$


thus, since $a^{n} x=0$ for $n \geq 2$,

$$
1=\|\exp (i t a) x\|=\|x+i \operatorname{tax}\| \text {. }
$$

Therefore $|t|\|a x\|-1 \leq \| x+i$ tax $\|=1$ for all $t \in \mathbb{R}$, hence $a x=0$.

Proposition 2.4. Suppose that $a, b \in \mathcal{A}, b$ is normal, $b=h+i k, h, k \in$ $H(\mathcal{A}), h k=k h, a b=\lambda b a$ and $\lambda \in \mathbb{R}$. Then

$$
a h=\lambda h a \text { and } a k=\lambda k a .
$$

Proof. Because of Proposition 2.1 we have $a b^{*}=\lambda b^{*} a$. Therefore $a\left(b+b^{*}\right)$ $=\lambda\left(b+b^{*}\right) a$ and $a\left(b-b^{*}\right)=\lambda\left(b-b^{*}\right) a$. Since $h=\frac{1}{2}\left(b+b^{*}\right)$ and $k=\frac{1}{2 i}\left(b-b^{*}\right)$, the result follows.

Now we are in a position to state the main result of this paper, a generalization of Theorem 1.1.

TheOREM 2.5. Let $a, b \in \mathcal{A}$ such that $a b=\lambda b a \neq 0, \lambda \in \mathbb{C}$.

(a) If $a \in H(\mathcal{A})$, then $\lambda \in \mathbb{R}$.

(b) If $a \in H(\mathcal{A})$ and $b$ is normal, then $\lambda \in\{-1,1\}$.

(c) If $a \in H(\mathcal{A})$ is positive and $b$ is normal then $\lambda=1$.

Proof. (a) follows from Corollary 2.2.

For the proof of (b) and (c) let $b=h+i k, h, k \in H(\mathcal{A})$ and $h k=k h$. Then, by (a) and Proposition 2.4, $\lambda \in \mathbb{R}, a h=\lambda h a$ and $a k=\lambda k a$. Since $a b \neq 0$, we have $a h \neq 0$ or $a k \neq 0$. Thus we can assume that $b$ is Hermitian.

(b) If $\lambda=1$, we are done. So assume that $\lambda \neq 1$. Then

$$
i(a b-b a)=i(\lambda b a-b a)=i(\lambda-1) b a \neq 0 .
$$

Proposition 1.4(d) shows that $i(\lambda-1) b a \in H(\mathcal{A})$, thus, by Proposition 1.4(e)

$$
|\lambda-1| r(b a)=r(i(\lambda-1) b a)=\|i(\lambda-1) b a\|=|\lambda-1|\|b a\| \neq 0,
$$

hence $r(b a) \neq 0$. Because of Proposition 1.3 we derive $|\lambda|=1$. Since $\lambda \in \mathbb{R} \backslash\{1\}, \lambda=-1$.

(c) From (b) we get $\lambda=1$ or $\lambda=-1$. Assume that $\lambda=-1$, thus $a b=-b a$. Then $b a b=-b^{2} a$ and $a b^{2}=-b a b$, hence

$$
b^{2} a=a b^{2}=-b a b .
$$

Since $\sigma\left(b^{2}\right) \subseteq[0, \infty)$ (Proposition 1.2(d)), $\sigma(a) \subseteq[0, \infty)$ and $a b^{2}=b^{2} a$, we derive from Proposition 1.2(c) that

$$
\sigma\left(a b^{2}\right) \subseteq \sigma(a) \sigma\left(b^{2}\right) \subseteq[0, \infty) .
$$

From $i(a b-b a)=2 i a b$ we see that $i a b \in H(\mathcal{A})$ (Proposition 1.4(d)).

Furthermore we have by (3) that

$$
i((i a b) b-b(i a b))=-a b^{2}+b a b=-2 a b^{2} .
$$


Use again Proposition 1.4(d) to get $a b^{2} \in H(\mathcal{A})$. It follows from (4) that $a b^{2}=-b a b$ is positive. Proposition 1.2(b) and (3) show that

$$
\sigma\left(a b^{2}\right) \backslash\{0\}=\sigma(-b(a b)) \backslash\{0\}=\sigma\left(-a b^{2}\right) \backslash\{0\},
$$

thus

$$
\sigma\left(a b^{2}\right)=\sigma\left(-a b^{2}\right)
$$

therefore, by (4), $\sigma\left(a b^{2}\right)=\{0\}$. Since $a b^{2} \in H(\mathcal{A})$, we derive from Proposition 1.4(e) that $\left\|a b^{2}\right\|=r\left(a b^{2}\right)=0$. Hence $b^{2} a=a b^{2}=0$. By Proposition 2.3 we obtain the contradiction $a b=-b a=0$. Thus $\lambda=1$.

Corollary 2.6. If $a \in H(\mathcal{A})$ is positive and $b \in \mathcal{A}$ is normal, then the following statements are equivalent:

(a) $a b=b a$;

(b) $a^{2} b=b a^{2}$.

Proof. The implication (a) $\Rightarrow(\mathrm{b})$ is clear.

(b) $\Rightarrow$ (a): Proposition 2.1 gives $a^{2} b^{*}=b^{*} a^{2}$. Let $b=h+i k$ with $h, k \in$ $H(\mathcal{A})$ and $h k=k h$. Then

$$
a^{2} h=a^{2} \frac{1}{2}\left(b+b^{*}\right)=\frac{1}{2}\left(b+b^{*}\right) a^{2}=h a^{2}
$$

and

$$
a^{2} k=a^{2} \frac{1}{2 i}\left(b-b^{*}\right)=\frac{1}{2 i}\left(b-b^{*}\right) a^{2}=k a^{2} .
$$

Thus, by (5),

$$
a(a h-h a)+(a h-h a) a=a^{2} h-a h a+a h a-h a^{2}=0 .
$$

Put $c=i(a h-h a)$. Then $c \in H(\mathcal{A})$ (Proposition 1.4(d)) and $a c=-c a$. Theorem 2.5(c) shows now that $a c=c a=0$. Thus $a(a h-h a)=0=$ $(a h-h a) a$. Now use the Kleinecke-Shirokov theorem ([4, Theorem 1.3.1]) to conclude that $r(a h-h a)=0$. Therefore $r(c)=0$, thus, since $c \in H(\mathcal{A})$, $c=0$. This gives $a h=h a$. A similar argument, use (6), shows that $a k=k a$. Hence $a b=a(h+i k)=(h+i k) a=b a$.

Corollary 2.7. If $a, b \in H(\mathcal{A})$ and $a$ is positive, then the following statements are equivalent:

(a) $a b=b a$;

(b) $a^{2} b=b a^{2}$;

(c) $a^{2}(a b-b a)=(a b-b a) a^{2}$.

Pro of. Because of Corollary 2.6 we only have to show that (c) implies (a). So suppose that (c) holds. Then, if $c=i(a b-b a)$, we have $c \in H(\mathcal{A})$ and $a^{2} c=c a^{2}$. By Corollary 2.6, $a c=c a$, thus $a(a b-b a)=(a b-b a) a$. Apply the Kleinecke-Shirokov theorem to get $r(c)=r(a b-b a)=0$. Proposition 1.4(e) shows now that $a b=b a$. 


\section{References}

[1] E. Berkson, H. R. Dowson and G. A. Elliot, On Fuglede's theorem and scalar-type operators, Bull. London Math. Soc. 4 (1972), 13-16.

[2] F. F. Bonsall and J.Duncan, Numerical Ranges of Operators on Normed Spaces and Elements of Normed Algebras, Cambridge University Press (1971).

[3] J. A. Brooke, P. Busch and B. Pearson, Commutativity up to a factor of bounded operators in complex Hilbert space, R. Soc. Lond. Proc. Ser. A, Math. Phys. Eng. Sci. A 458 (2002), 109-118.

[4] C. R. Putnam, Commutation Properties of Hilbert Space Operators, Springer (1967).

[5] W. Rudin, Functional Analysis, Mc Graw-Hill (1973).

[6] J. Yang and H. Du A note an commutativity up to a factor of bounded operators, Proc. Amer. Math. Soc. 132 (2004), 1713-1720.

MATHEMATISCHES INSTITUT I

UNIVERSITÄT KARLSRUHE

D-76128 KARLSRUHE, GERMANY

e-mail: christoph.schmoegerOmath.uni-karlsruhe.de

Received April 13, 2004. 